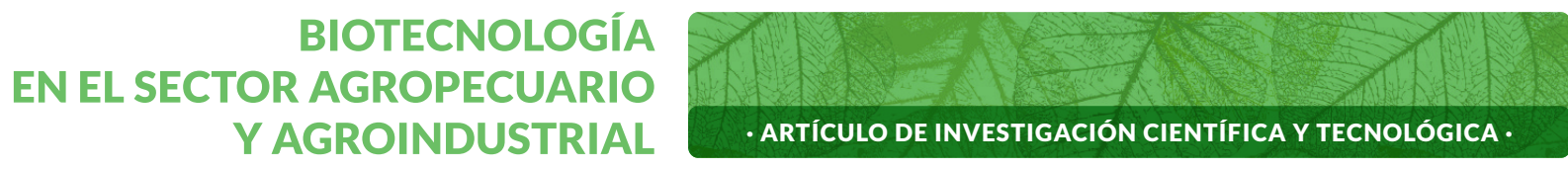

Vol. 19 No 2 • Julio - Diciembre 2021 • ISSN - 1692-3561 • ISSN-e 1909-9959 • https://doi.org/10.18684/bsaa.v19.n2.2021.1363

\title{
Cama de aves de corral un factor importante en la seguridad alimentaria*
}

\section{Poultry litter an important factor in food safety}

OSPINA-BARRERO, MARIA-ALEJANDRA ${ }^{1}$; BORSOI, ANDERLISE ${ }^{2}$;

PEÑUELA-SIERRA, LINA-MARIA ${ }^{3}$; VARON-LOPEZ, MARYEIMY ${ }^{4}$

\section{RESUMEN}

La cama es un componente esencial en la producción de aves de corral, ya que habitan sobre este material la mayor parte de su ciclo productivo, por consiguiente, en ella se puede encontrar heces, bacterias entéricas, patógenos de importancia zoonótica y/o de transmisión alimentaria, plaguicidas y antimicrobianos, los cuales pueden afectar la sanidad

Historial del artículo

Recibido para evaluación: 5 de Febrero 2020

Aprobado para publicación: 27 de Febrero 2021

* Título del proyecto de origen: "Respuesta de la microbiota de las camas de pollo de engorde, a la invasión de Salmonella Enteritidis”. Financiación: Minciencias. Culminación: 30 de Mayo de 2022.

1 Universidad del Tolima, Facultad de Ciencias, Grupo de Investigación Genética y Biotecnología Vegetal y Microbiana de la Universidad del Tolima. Magister en Medicina Veterinaria. Ibagué, Colombia. https://orcid.org/0000-0002-0806-3677

2 Universidad Tuiuti de Paraná, FACBS. Doctor en Ciencias Veterinarias. Curitiba, Brasil. https://orcid.org/0000-0003-43015686

3 Universidad del Tolima, Facultad de Medicina Veterinaria y Zootecnia, Grupo de Investigación Sistemas Agroforestales Pecuarios. Doctor en Zootecnia. Ibagué, Colombia. https://orcid.org/0000-0002-0483-2176

4 Universidad del Tolima, Facultad de Ciencias, Grupo de Investigación Genética y Biotecnología Vegetal y Microbiana. Doctor en Ciencias. Ibagué, Colombia. https://orcid.org/0000-0003-1125-2329

Correspondencia: maospina@ut.edu.co

Cómo citar este artículo: OSPINA-BARRERO, MARIA-ALEJANDRA; BORSOI, ANDERLISE; PEÑUELA-SIERRA, LINA-MARIA; VARON-LOPEZ, MARYEIMY. Cama de aves de corral un factor importante en la seguridad alimentaria. Revista Biotecnología en el Sector Agropecuario y Agroindustrial, v. 19, n. 2, 2021, p. 234-250. Doi: https://doi.org/10.18684/bsaa.v19.n2.2021.1451 
de las aves. Después del ciclo de producción avícola, la cama es comúnmente utilizada como fertilizante orgánico para mejorar la calidad de los suelos y los cultivos. A pesar de su amplio uso en la avicultura y agricultura, se conoce poco acerca de su importancia en la seguridad alimentaria, por lo cual, la presente revisión discute acerca de los patógenos y contaminantes presentes en la cama y sus riesgos para la seguridad alimentaria, además las prácticas de manejo y tratamientos más adecuados. Información que será útil para maximizar los usos de la cama y los desechos avícolas, para que estos no representen un peligro para las personas, animales y el medio ambiente.

\section{ABSTRACT}

The litter is an essential component in poultry production, as the birds inhabit this material for most of their production cycle. As a result, located in the litter is feces, enteric bacteria, pathogens of zoonotic importance and / or foodborne pathogens, pesticides and antimicrobials, which affect the health of birds. After the poultry production cycle, litter is commonly used as an organic fertilizer to improve the quality of the soil and of crops. Despite its wide use in poultry and agriculture, little is known about its importance in food security. The present study provides more information about litter-borne pathogens, the risks associated with contaminants and zoonotic diseases in the waste generated from poultry, management practices and treatments. It is essential to consider the impact of the litter because not doing so represents a danger to humans, animals, and the environment.

\section{INTRODUCCIÓN}

La avicultura es uno de los sectores pecuarios más importantes en el aporte de proteína de origen animal en Colombia, reflejado en el consumo per cápita de huevo ( 294 unidades) y de carne de pollo (34 kg), alcanzando una producción de 14.606 millones de unidades y de 1.624.000 toneladas respectivamente, para el año 2018 según la Federación Nacional de Avicultores (FENAVI, 2019). Estos productos avícolas se caracterizan por tener un gran valor nutricional ya que aportan aminoácidos, vitaminas y minerales, fundamentales en la base de la alimentación (FENAVI, 2019).

En la producción avícola, diferentes factores influyen en el bienestar, desempeño y salud de los animales, entre estos el sustrato de la cama donde habitan los pollos de engorde (Dunlop et al., 2016). Este material, habitualmente contiene residuos de antimicrobianos, amoniaco, plaguicidas y patógenos zoonoticos, los cuales representan un peligro para las aves (Brooks et al., 2016). Después de su uso en la avicultura, la cama es utilizada como fertilizante, muchas veces sin un tratamiento correcto, contaminando el medio ambiente e incluso los vegetales cultivados para consumo humano, lo que podría desencadenar brotes de Enfermedades Transmitidas por Alimentos (ETA) (Agostinho et al., 2020).

\section{PALABRAS CLAVE:}

Agricultura; Antimicrobianos; Avicultura; Cama de aves; Desechos avícolas; Fertilizante; Gallinaza; Patógenos; Pollinaza; Seguridad alimentaria.

\section{KEY WORDS:}

Agriculture; Antimicrobials; Poultry; Poultry litter; Poultry waste; Fertilizer; Chicken manure; Pathogens; Poultry manure; Food safety. 
Por tal razón, es necesario prestar atención a los factores involucrados en el sistema de producción avícola, siendo el manejo de la cama uno de los factores más importantes (Brooks et al., 2016); teniendo en cuenta que patógenos como Salmonella spp. y Campylobacter spp. Son frecuentemente aislados de este material, los cuales son agentes etiológicos de la salmonelosis y campilobacteriosis en humanos y comúnmente son asociadas con el consumo de productos avícolas (Kagambèga et al., 2018). Así mismo, estudios epidemiológicos relacionados a estas enfermedades en aves de corral, junto con las altas incidencias de ETA, indican que las medidas de control de estas enfermedades son insuficientes (Premarathne et al., 2017; Castañeda-Salazar et al., 2018).

Por consiguiente, el objetivo de la presente revisión, es discutir la importancia de la cama de aves de corral en la seguridad alimentaria y sus riesgos como fuente de contaminantes, con el fin de generar un referente en Colombia, para que se realicen controles sobre este material y se asegure su calidad e inocuidad y así puedan ser usados en la avicultura y la agricultura.

\section{DESARROLLO DEL TEMA}

\section{Propiedades de la cama}

La cama es el material que cubre el piso del galpón, la cual contribuye al bienestar y el desarrollo adecuado a las aves, se utiliza para reducir fluctuaciones de temperatura, absorber el agua, incorporar heces, orina y plumas (Bolan et al., 2010; Dunlop et al., 2016). Además, interfiere en la sanidad del galpón, debido al hábito de consumo de cama y el contacto directo de los animales con este material (Wang et al., 2016; Stojcic et al., 2016). Entre los insumos aprovechados como sustrato para la cama se encuentran: la cascarilla de café, mazorca de maíz molida, viruta de madera, cascarilla de fríjol, paja de heno, cáscara de papa, cáscara de coco, cáscara de maní y cascarilla de arroz (Wang et al., 2016; Stojcic et al., 2016).

Después de la cría de un lote de aves de corral, la cama se composta junto con las excretas y bacterias entéricas de los animales, residuos de la dieta, plumas e insectos (Wang et al., 2016); dando origen a una combinación rica en nitrógeno, fosforo y potasio, nutrientes importantes para la multiplicación de los microrganismos (Paterlini et al., 2017). En un lote de pollo de engorde se producen aproximadamente $6,9 \mathrm{~kg}$ de pollinaza por cada $1000 \mathrm{~kg}$ de peso vivo por día (Bolan et al., 2010), obligando al sector agrícola a buscar alternativas sostenibles para el uso de estos desechos. Por esta razón, la reutilización de la cama en pollos de engorde es una práctica común en la avicultura moderna, debido a su alto costo, escasez y material contaminante (Vaz et al., 2017). Sin embargo, este reúso causa un aumento en la humedad, $\mathrm{pH}$, amoníaco y diversidad de microorganismos principalmente patógenos, los cuales pueden transmitirse de un ciclo productivo a otro (Wang et al., 2016; Vaz et al., 2017).

\section{Patógenos de transmisión alimentaria en la cama}

Entre los microorganismos aislados en camas de aves de corral se encuentran patógenos productores de ETA tales como: Salmonella spp, Streptococcus, Campylo- bacter spp, Corynebacterium, Listeria monocytogenes, Globicatella, Bordetella, Es- cherichia coli, Clostridium perfringens, Clostridium botulinum y Staphylococcus aureus (Bolan et al., 2010) como se muestra en el cuadro 1.

Estos patógenos pueden transmitirse de un ciclo productivo a otro por diversos factores como son el reúso de la cama sin tratamientos adecuados por varios lotes consecutivos, el hábito de consumo de cama por las aves y el contacto directo de los animales con este material, pudiendo provocar un aumento de la trasmisión de estos microorganismos dentro de la cadena alimentaria hasta el consumidor (Dunlop et al., 2016; Wang et al., 2016). Ejemplo de ello son las relaciones fenotípicas y genéticas encontradas entre $S$. Enteritidis aislada de humanos con gastroenteritis y aves de corral en el Tolima (Fandiño and Verjan, 2019). 
Así mismo, los brotes de ETA presentados en Colombia en los últimos tres años (2017- 2019), pueden estar influenciados por la contaminación de los pollos de engorde en las granjas, debido a que los productos de origen animal (cárnicos, principalmente el pollo) fueron los alimentos mayormente involucrados, siendo los agentes etiológicos hallados con mayor frecuencia: Escherichia coli, Salmonella spp, Staphylococus aureus y Coliformes fecales (patógenos reportados en cama de aves de corral) Instituto Nacional de Salud (INS 2017, 2018, 2019).

Cuadro 1. Patógenos en cama y heces de aves de corral, fuente de infección para humanos y síntomas de las infecciones.

\begin{tabular}{|c|c|c|c|}
\hline Patógeno & Fuente de infección & Síntomas en humanos & Referencias \\
\hline Campylobacter spp. & $\begin{array}{l}\text { Consumo de productos avícolas } \\
\text { contaminados con Campylobacter spp., } \\
\text { provenientes de lotes de pollos con camas } \\
\text { reutilizadas. }\end{array}$ & $\begin{array}{l}\text { Enfermedad inflamatoria intestinal, esófago } \\
\text { de Barrett y cáncer colorrectal, bacteremia, } \\
\text { infecciones pulmonares, abscesos cerebra- } \\
\text { les, meningitis y artritis reactiva. }\end{array}$ & $\begin{array}{l}\text { (Ahmed et al., } \\
\text { 2013) }\end{array}$ \\
\hline Salmonella spp. & $\begin{array}{l}\text { Consumo de pollo contaminado con } \\
\text { Salmonella spp., mediante la ingestión de } \\
\text { cama infectada en el periodo de retiro de } \\
\text { alimento, antes de ser enviados a la planta } \\
\text { de beneficio }\end{array}$ & $\begin{array}{l}\text { Inflamación intestinal, diarrea mucopuru- } \\
\text { lenta o sanguinolenta, acompañada de } \\
\text { fiebre, vómitos y calambres abdominales. }\end{array}$ & $\begin{array}{l}\text { (Andoh et al., } \\
\text { 2017; Kagambè- } \\
\text { ga et al., 2018) }\end{array}$ \\
\hline Escherichia coli & $\begin{array}{l}\text { Contacto con cama de aves de corral } \\
\text { contaminada o consumo de productos } \\
\text { avícolas contaminados. }\end{array}$ & $\begin{array}{l}\text { Diarrea, colitis hemorrágica y síndrome } \\
\text { urémico hemolítico }\end{array}$ & $\begin{array}{l}\text { (Shecho et al., } \\
\text { 2017; Bell-cohn } \\
\text { et al., 2019) }\end{array}$ \\
\hline $\begin{array}{l}\text { Staphylococcus } \\
\text { aureus }\end{array}$ & $\begin{array}{l}\text { Infecta a los humanos a través del aire, agua } \\
\text { y granjas agrícolas donde el estiércol de aves } \\
\text { es utilizado como fertilizante orgánico. }\end{array}$ & $\begin{array}{l}\text { Infecciones de piel y tejidos blandos, absce- } \\
\text { sos, conjuntivitis, osteomielitis, me- } \\
\text { ningitis, sepsis, endocarditis o neumonía. }\end{array}$ & $\begin{array}{l}\text { (Kobayashi et } \\
\text { al., 2015; Smith, } \\
\text { 2015) }\end{array}$ \\
\hline $\begin{array}{l}\text { Clostridium botu- } \\
\text { linum }\end{array}$ & $\begin{array}{l}\text { Consumo de carne bovina contaminada } \\
\text { por esporas de la bacteria en suelos ferti- } \\
\text { lizados con gallinaza o pollinaza. }\end{array}$ & $\begin{array}{l}\text { Insuficiencia respiratoria, problemas diges- } \\
\text { tivos, alteraciones neurológicas y en algunos } \\
\text { casos muerte súbita. }\end{array}$ & $\begin{array}{l}\text { (Pignata et al., } \\
\text { 2019) }\end{array}$ \\
\hline $\begin{array}{l}\text { Clostridium } \\
\text { perfringens }\end{array}$ & $\begin{array}{l}\text { Consumo de alimentos, agua, suelo conta- } \\
\text { minados con cama de aves de corral. }\end{array}$ & $\begin{array}{l}\text { Diarrea, dolor abdominal, muertes oca- } \\
\text { sionales en adultos mayores y personas } \\
\text { hospitalizadas }\end{array}$ & $\begin{array}{l}\text { (Labbe and June- } \\
\text { ja, 2017 Milanov } \\
\text { et al., 2019) }\end{array}$ \\
\hline $\begin{array}{l}\text { Listeria monocyto- } \\
\text { genes }\end{array}$ & $\begin{array}{l}\text { Ingestión de agua y vegetales contamina- } \\
\text { dos después de la aplicación de cama de } \\
\text { aves de corral en el suelo. } \\
\text { Consumo de aves y carne bovina contami- } \\
\text { nada con la bacteria. }\end{array}$ & $\begin{array}{l}\text { Gastroenteritis leve a severa, afecta princi- } \\
\text { palmente a niños, personas inmuno- com- } \\
\text { prometidas y puede provocar septice- mia, } \\
\text { meningitis e infección fetal o aborto. }\end{array}$ & $\begin{array}{l}\text { (Dahshan, 2016; } \\
\text { Radoshevichand } \\
\text { Cossart, 2017) }\end{array}$ \\
\hline Penicillium & $\begin{array}{l}\text { Trabajadores inhalan polvo al esparcir la } \\
\text { cama de aves de corral contaminada en } \\
\text { los galpones y en el campo. }\end{array}$ & $\begin{array}{l}\text { Estos hongos son fuente de aflatoxinas } \\
\text { relacionadas con diferentes tipos de cáncer } \\
\text { entre estos el de hígado. }\end{array}$ & $\begin{array}{l}\text { (Viegas et al., } \\
\text { 2015; Ramírez } \\
\text { et al., 2017) }\end{array}$ \\
\hline Fusarium & $\begin{array}{l}\text { Inhalación de partículas al esparcir la } \\
\text { cama de aves de corral contaminada en } \\
\text { los galpones y en suelos. }\end{array}$ & $\begin{array}{l}\text { Onicomicosis, queratitis, sinusitis, neumonía } \\
\text { en pacientes inmunodeprimidos. }\end{array}$ & $\begin{array}{l}\text { (Martínez et al., } \\
\text { 2014; Gómez et } \\
\text { al., 2018) }\end{array}$ \\
\hline $\begin{array}{l}\text { Histoplasma capsu- } \\
\text { latum }\end{array}$ & $\begin{array}{l}\text { Trabajadores inhalan polvo al esparcir } \\
\text { la cama de aves de corral contaminada } \\
\text { en los galpones o por manipulación de } \\
\text { fertilizantes orgánicos. }\end{array}$ & $\begin{array}{l}\text { Histoplasmosis, afecciones respiratorias y } \\
\text { alteraciones neurológicas. }\end{array}$ & $\begin{array}{l}\text { (Gómez et al., } \\
\text { 2018; Riddell } \\
\text { and Wheat, } \\
\text { 2019) }\end{array}$ \\
\hline Cryptosporidium & $\begin{array}{l}\text { Beber agua contaminada, consumo de } \\
\text { productos frescos que han sido fertiliza- } \\
\text { dos con estiércol de aves de corral. }\end{array}$ & $\begin{array}{l}\text { Dolor abdominal, fiebre, vómitos, malab- } \\
\text { sorción y diarrea. Los pacientes con VIH } \\
\text { (no tratados con terapia antirre- troviral), } \\
\text { a menudo sufren de diarrea intratable, que } \\
\text { puede ser mortal. }\end{array}$ & $\begin{array}{l}\text { (Ryan et al., } \\
\text { 2016; Vermeu- } \\
\text { len et al., 2017) }\end{array}$ \\
\hline $\begin{array}{l}\text { Virus de la influenza } \\
\text { aviar tipo A }\end{array}$ & $\begin{array}{l}\text { Cama de aves de corral utilizada como } \\
\text { fertilizante en jardinería con heces conta- } \\
\text { minadas con el virus H5N1. }\end{array}$ & $\begin{array}{l}\text { Fiebre, dolor de garganta y cabeza, tos, } \\
\text { dificultad respiratoria y muerte. }\end{array}$ & $\begin{array}{l}\text { (Kandun et al., } \\
\text { 2010) }\end{array}$ \\
\hline $\begin{array}{l}\text { Virus de la enferme- } \\
\text { dad de Newcastle }\end{array}$ & $\begin{array}{l}\text { Mal manejo del estiércol de aves de } \\
\text { corral. }\end{array}$ & Conjuntivitis & $\begin{array}{l}\text { (Guan et al., } \\
\text { 2009) }\end{array}$ \\
\hline
\end{tabular}

Otros factores que pueden influir en la transferencia de patógenos presentes en las camas a los humanos es el uso de la gallinaza o pollinaza como acondicionadores de suelos sin un tratamiento correcto, dado que los mi- 
croorganismos al llegar a este ambiente pueden contaminar vegetales, como la lechuga, espinacas y tomate, que al consumirse de forma cruda representan un mayor riesgo (Fang et al., 2015; Shah et al., 2019).

Salmonella spp. y Campylobacter spp. son los microorganismos de mayor distribución en camas de aves a nivel mundial, su supervivencia depende del serotipo, manipulación de la cama, edad de las aves, área geográfica y época del año (Li et al., 2007) (cuadro 2). Salmonella spp. se puede encontrar en cama de pollo de engorde de 1,1 a $4 \times 10^{5} \mathrm{UFC} / \mathrm{g}$, (Unidades Formadoras de Colonias); con un porcentaje de aislamiento variable, con valores del 50 \% en Mumbai, India; del 53,33 \% Gobernación de Beni Suef (Ibrahim et al., 2013); y del 72 \% en Kyushu, Japón (Yamazaki et al., 2016).

Cuadro 2. Tiempo de supervivencia de patógenos de transmisión alimentaria en cama y heces de aves corral

\begin{tabular}{|c|c|c|c|}
\hline Cama o heces & Serotipo & $\begin{array}{c}\text { Tiempo de } \\
\text { supervivencia }\end{array}$ & Referencia \\
\hline Cama de pollo $70^{\circ} \mathrm{C}$ a $20-30-40-50 \%$ de humedad & Salmonella spp. & $>6$ horas & (Chen et al., 2013) \\
\hline Cama de pollo nueva $50^{\circ} \mathrm{C}$ & Salmonella spp. & $<50$ horas & (Biswas et al., 2019) \\
\hline Cama de pollo nueva $60^{\circ} \mathrm{C}$ & Salmonella spp. & $<1$ hora & (Biswas et al., 2019) \\
\hline Cama de pollo nueva $50^{\circ} \mathrm{C}$ & Escherichia coli & $>66$ horas & (Biswas et al., 2019) \\
\hline Cama de pollo nueva $60^{\circ} \mathrm{C}$ & Escherichia coli & $<3$ horas & (Biswas et al., 2019) \\
\hline $\begin{array}{l}\text { Cama de pollo almacenada profundidad } \\
(50 \mathrm{~cm}) \text { en invierno }\end{array}$ & Escherichia coli (BLEE) & 48 horas & (Siller et al., 2020) \\
\hline $\begin{array}{l}\text { Superficie de cama de pollo almacenada } \\
\text { en invierno }\end{array}$ & Escherichia coli (BLEE) & $>72$ horas & (Siller et al., 2020) \\
\hline Superficie de cama de pollo almacenada en verano & Escherichia coli (BLEE) & $>72$ horas & (Chen and Jiang, 2014) \\
\hline $\begin{array}{l}\text { Heces de gallina ponedora inoculadas artificialmente } \\
20^{\circ} \mathrm{C} \text { a } 40-60 \% \text { de humedad relativa }\end{array}$ & C. jejuni & $72-96$ horas & (Ahmed et al., 2013) \\
\hline Heces de gallina ponedora & C. jejuni & $120-144$ horas & (Bell-cohn et al., 2019) \\
\hline Heces de pollo inoculadas en microaerofilia & C. jejuni & 72 horas & (Smith et al., 2016) \\
\hline Heces de pollo inoculados en condiciones aerobias & C. jejuni & 48 horas & (Smith et al., 2016) \\
\hline Cama de pollo & C. jejuni & 4 horas & (Smith et al., 2016) \\
\hline
\end{tabular}

Otros de los contaminantes encontrados con frecuencia en la cama son los residuos de antibióticos, utilizados en la avicultura para prevenir y tratar enfermedades infecciosas o como promotores del crecimiento animal (APC) (Xiong et al., 2018), los cuales se suministran en dosis subterapéuticas en la dieta de las aves, con el fin de aumentar la tasa de crecimiento, rendimiento productivo y proteger la salud de los animales al modificar el sistema inmunológico (Gonzalez and Angeles, 2017).

Entre los APC manejados en la industria avícola se encuentran: la enramicina, bacitracina de zinc, virginiamicina, avilamicina, entre otros, los cuales no se absorben completamente en el intestino de los pollos y hasta un $90 \%$ de la dosis administrada puede excretarse en las heces (Yang et al., 2017) (Cuadro 3); motivo por el cual se han detectado trazas de antibióticos y bacterias resistentes a los antibióticos en suelos y cultivos fertilizados con gallinaza o pollinaza, lo que podría generar un riesgo potencial para la salud humana (Kassem et al., 2017).

En este sentido, a nivel mundial se han desarrollado investigaciones con el fin de determinar los mecanismos y perfil de resistencia bacteriana en camas de aves de corral, es así, como Nandi et al. (2004) cuantificaron genes de resistencia a los antibióticos e integrones y encontraron que el reservorio principal para los integrones de Clase 1, son las bacterias Gram-positivas, las cuales constituyen más del 85 \% de la comunidad microbiana presente en camas de aves. Islam et al. (2008) investigaron la relación entre el uso de antibióticos y el desarrollo de resistencia en cepas de E. coli aisladas de cama, en Chittagong, Bangladesh, donde encontraron que todos los aislados eran sensibles a imipenem y a su vez expresaron resistencia a múltiples antibióticos, como, ciprofloxacina (30\%), gentamicina (50\%), eritromicina (66,6\%), tetraciclina (96,6\%), penicilina (100\%), y cloranfenicol (100\%). Los investigadores concluyeron que los aislados de E. coli multirresistentes y los plásmidos que contie- 
nen genes resistentes a múltiples antibióticos están presentes en las granjas avícolas y las aves pueden actuar como una posible fuente de transferencia de estos estos patógenos y sus genes a humanos.

Cuadro 3. Nivel de excreción de antibióticos en heces de pollos.

\begin{tabular}{|l|l|l|}
\hline \multicolumn{1}{|c|}{ Antibiótico } & \multicolumn{1}{|c|}{ Porcentaje } & \multirow{2}{*}{ Referencia } \\
\hline Tetraciclinas & $75-80 \%$ & \multirow{2}{*}{ (Kumar et al., 2004) } \\
\hline Lincosamidas & $60 \%$ & \\
\hline Macrólidos & $50-90 \%$ & \\
\cline { 1 - 1 } $\begin{array}{l}\text { Penicilina Salinomicina Bacitracina } \\
\text { Clortetraciclina Virginiamicina y } \\
\text { Monensina }\end{array}$ & $3-60 \%$ & \\
\hline
\end{tabular}

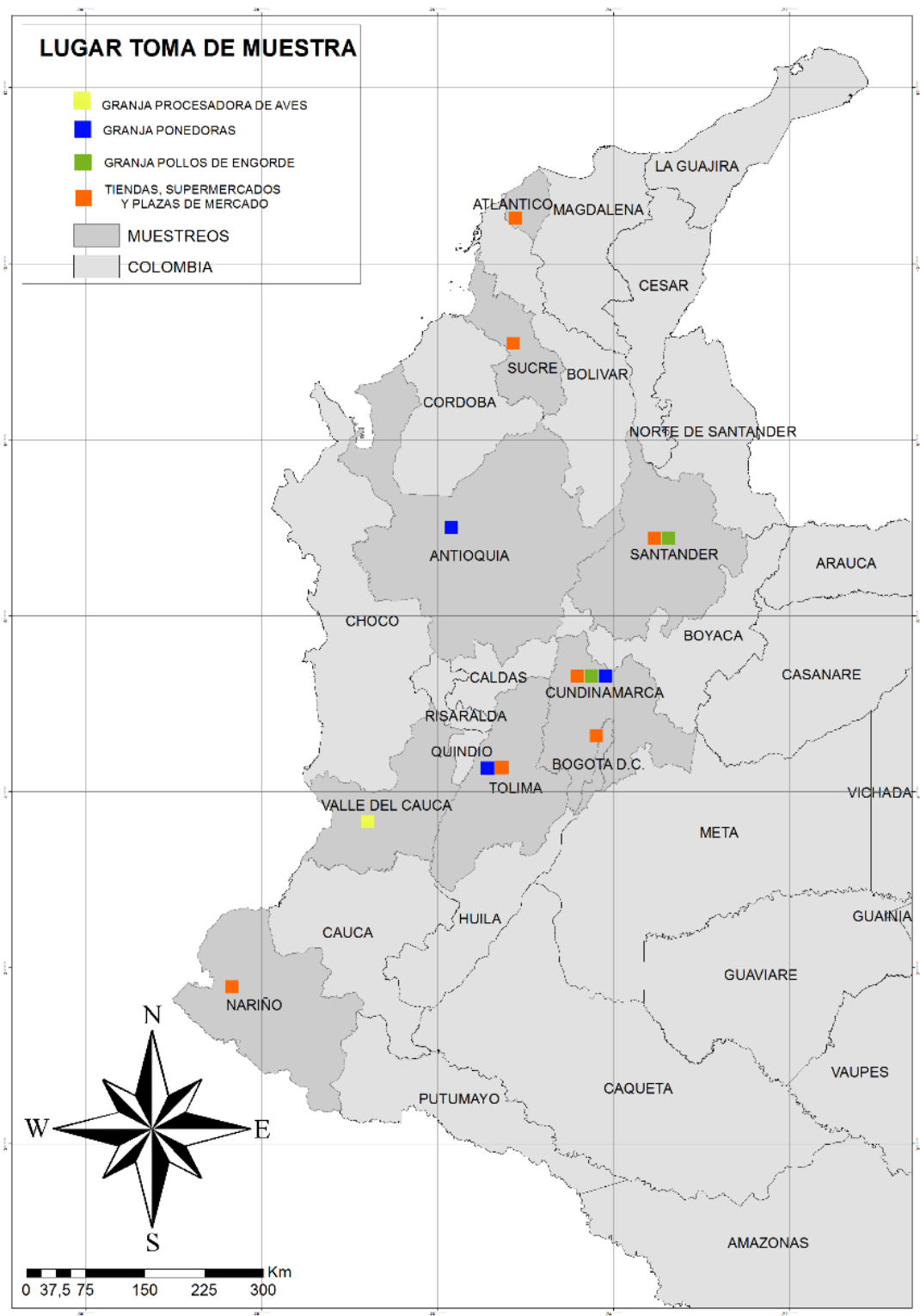

Figura 1. Departamentos y tipos de muestras dentro de la cadena avícola en los cuales se aislado Salmonella y Campylobacter 


\begin{tabular}{|c|c|c|c|c|c|}
\hline $\begin{array}{l}\text { Año del } \\
\text { estúdio }\end{array}$ & Departamento & $\begin{array}{l}\text { Lugar toma } \\
\text { de muestra }\end{array}$ & Tipo de muestra & Serotipos & Referencia \\
\hline 1985 & Valle del Cauca & $\begin{array}{l}\text { Granja procesado- } \\
\text { ra de aves }\end{array}$ & $\begin{array}{l}\text { Canal de pollo y } \\
\text { ciegos }\end{array}$ & C. jejuni & $\begin{array}{l}\text { (Carmona, } \\
\text { 1985) }\end{array}$ \\
\hline \multirow[b]{3}{*}{2004} & \multirow[b]{3}{*}{ Antioquia } & \multirow[b]{3}{*}{ Granja ponedoras } & Hisopos cloacales & S. Enteritidis & \multirow{3}{*}{$\begin{array}{l}\text { (Buitrago et } \\
\text { al., 2006) }\end{array}$} \\
\hline & & & $\begin{array}{l}\text { Hisopos cloacales } \\
\text { y cama }\end{array}$ & S. Infantis & \\
\hline & & & $\begin{array}{l}\text { Tejidos, corazón, } \\
\text { hígado, vesicula, } \\
\text { ovario, oviducto, } \\
\text { tonsila cecal }\end{array}$ & $\begin{array}{l}\text { S. Enteritidis } \\
\text { S. Infantis } \\
\text { S. Derby }\end{array}$ & \\
\hline 2009 & Cundinamarca & Granja ponedoras & $\begin{array}{l}\text { Hisopos de arrastre } \\
\text { (cama) }\end{array}$ & S. Yoruba & $\begin{array}{l}\text { (Pulido et al., } \\
\text { 2014) }\end{array}$ \\
\hline $\begin{array}{l}2008- \\
2010\end{array}$ & $\begin{array}{l}\text { Cundinamarca- } \\
\text { Santander }\end{array}$ & $\begin{array}{l}\text { Granja pollos } \\
\text { engorde }\end{array}$ & $\begin{array}{l}\text { Hisopos de arrastre } \\
\text { (cama) y muestras } \\
\text { fecales }\end{array}$ & S. Paratyphi S. Heidelberg & $\begin{array}{l}\text { (Donado et } \\
\text { al., 2012) }\end{array}$ \\
\hline $\begin{array}{l}2010- \\
2011\end{array}$ & $\begin{array}{l}\text { Sucre Santander } \\
\text { Tolima Cundi- } \\
\text { namarca Nariño } \\
\text { Atlantico }\end{array}$ & $\begin{array}{l}\text { Tiendas, supermer- } \\
\text { cados }\end{array}$ & Canales de pollo & $\begin{array}{l}\text { S. Paratyphi B dT S. Heidelberg S. } \\
\text { Anatum S. Enteritidis S. Derby S. Typhi- } \\
\text { murium S. Albany S. Bardo Braenderup } \\
\text { S. Kentucky S. Essen S. Mbandaka S. } \\
\text { Bareilly S. Tokoin S. Cuckmere S. Hogh- } \\
\text { ton S. Uganda S. Hillingdon S. Hoboken } \\
\text { S. Isangui S. Manhattan S. Virchow } \\
\text { S.Stanley S. Muenchen S.Senftenber } \\
\text { S.Wagenia S. Yovokome }\end{array}$ & $\begin{array}{l}\text { (Donado et } \\
\text { al., 2014) }\end{array}$ \\
\hline 2013 & Tolima & Granja ponedoras & Zapatones (cama) & S. Shannon S. Enteritidis & $\begin{array}{l}\text { (Rodríguez } \\
\text { et al., 2015) }\end{array}$ \\
\hline 2014 & Tolima (Ibagué) & $\begin{array}{l}\text { Tiendas, supermer- } \\
\text { cados }\end{array}$ & Canales de pollo & $\begin{array}{l}\text { S. Paratyphi B S. Hvittingfoss S. } \\
\text { Newport S. Bovismorbificans S. } \\
\text { Muenster S. Typhimurium S. Heidelberg } \\
\text { S.Braenderup S. Skansen S. Budapest S. } \\
\text { Manhattan S. Othmarschen S. Schwar- } \\
\text { zengrund }\end{array}$ & $\begin{array}{l}\text { (Rodríguez } \\
\text { JM et al., } \\
\text { 2015) }\end{array}$ \\
\hline 2015 & $\begin{array}{l}\text { Bogotá (Cundina- } \\
\text { marca) }\end{array}$ & $\begin{array}{l}\text { Tiendas y plazas de } \\
\text { mercado }\end{array}$ & Pechugas de pollo & $\begin{array}{l}\text { Salmonella spp. S. enterica group IIIb S. } \\
\text { Virchow S.Bredeney S. Anatum }\end{array}$ & $\begin{array}{l}\text { (Castañe- } \\
\text { da-Salazar } \\
\text { et al., 2018) }\end{array}$ \\
\hline 2016 & Santander & $\begin{array}{l}\text { Granja pollos } \\
\text { engorde }\end{array}$ & Hisopos cloacales & S. Heidelberg & $\begin{array}{l}\text { (Castro et } \\
\text { al., 2019) }\end{array}$ \\
\hline
\end{tabular}

Según la Organización Mundial de la Salud (OMS, 2014), la resistencia antimicrobiana se ha transformado en un importante problema para la salud pública, principalmente cuando se origina frente a antimicrobianos de primera línea de elección, ya que la principal amenaza es que a corto plazo no existirán antimicrobianos para tratar este tipo de bacterias resistentes en la medicina humana; aproximadamente 7,000,000 muertes por año en el mundo se han asociado a esta problemática (Xie et al., 2018).

Adicional a los contaminantes mencionados, cuando las camas son sometidas a tratamientos térmicos inadecuados, se genera dioxinas como subproducto del uso de antibióticos y anticoccidiales clorados como el amprolio, diclazuril y clopidol (Kyakuwaire et al., 2019; Liu et al., 2019). Las cuales tienen un efecto disruptor endocrino, carcinogénico y mutagénico en animales y humanos (Kyakuwaire et al., 2019; Conesa et al., 2019). Por esta razón es importante tener en cuenta la temperatura y el tiempo de incineración de la cama, cuando se va utilizar como fuente de energía o para su eliminación.

\section{Manejo y tratamientos de la cama}

En las buenas prácticas de manejo de la cama en los sistemas de producción avícola, se deben considerar la elección del material que la constituyen, tratamiento y condiciones en las cuales debe ser retirada del galpón 
al final del ciclo productivo, ya que al tener en cuenta estos factores se contribuye a reducir los malos olores y las características favorables para la supervivencia de patógenos en este ambiente. Con base en lo anterior, en el presente estudio se realizó una encuesta a profesionales directores de granjas productoras de material genético registradas ante el Instituto Colombiano Agropecuario (ICA) con registro vigente al año 2020, a fin de evidenciar las prácticas de manejo de la cama en este sistema de producción. Se encontraron 148 granjas registradas, de las cuales fue posible obtener información de 101. Se elaboró una encuesta con cinco preguntas de tipo cerrada y una de tipo abierto, la cuales fueron enviadas por correo electrónico, datos que fueron tabulados para realizar un análisis descriptivo y porcentual de los mismos.

Se evidenció que la mayoría se encuentran en el Valle del Cauca (53\%) y Cundinamarca (31\%) y en una menor proporción en los departamentos de Tolima (7\%), Atlántico $6 \%$ ) y Santander (3 \%). Se logró constatar que el material predominante utilizado como cama fue viruta de madera (62\%) y cascarilla de arroz (38 \%), lo cual dependía principalmente de la oferta de estos subproductos en la región. Es de resaltar que la viruta de madera resulta ser una elección que favorece la inocuidad de la cama, dado que la madera contiene metabolitos con efecto antimicrobiano (Vainio et al., 2017), además posee una buena capacidad de absorción de la humedad, disminuyendo la supervivencia de patógenos en este material (Farhadi, 2014).

Así mismo, se encontró que antes del ciclo productivo la mayoría de las granjas (98 \%) realizan algún tipo de tratamiento de la cama, siendo el uso de bactericidas y antifúngicos el de mayor elección, seguido del uso de cal y calor (figura 2). Este tipo de procedimientos reduce el crecimiento de patógenos (Sheffield et al., 2018) y con ello disminuye el riego de infección en las aves.

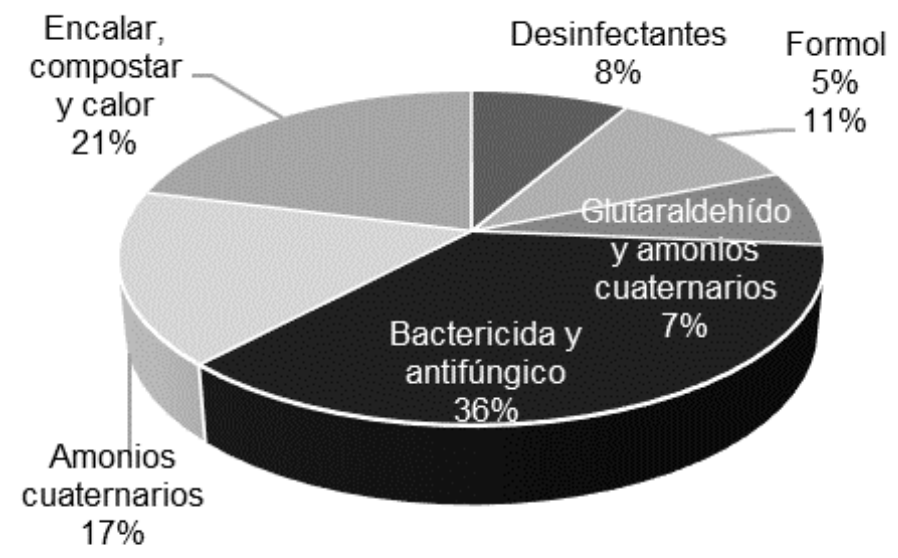

Figura 2. Tratamiento de la cama antes de su uso.

Por otro lado, también se encontró que terminado el ciclo de producción, el 79 \% de las granjas realizaban sanitización como tratamiento final de las camas y el restante compostaje, para realizar un seguimiento del control microbiológico de las camas el 74 \% de las granjas realizaban este tipo de análisis, tanto en las camas antes de la entrada de las aves, durante el ciclo productivo o después del tratamiento final. Otro de los resultados encontrados a través de este estudio, fue que el $88 \%$ de las granjas vendían la gallinaza y el $22 \%$ la utilizaban como abono y fertilizante, indicando que todo el material obtenido al final del proceso productivo tenía un uso posterior. Esto indica que realmente es fundamental conocer el estado microbiológico de las camas, para poder plantear y tomar medidas que contribuyan a la reducción de la carga de patógenos y por lo tanto evitar infección en las aves. Igualmente, es necesario resaltar que así el material de la cama sea sometido a algún tipo de tratamiento es necesario realizar de nuevo análisis microbiológicos que permitan verificar la eficacia de los mismos, ya que a pesar de que el compostaje es un método adecuado para eliminar patógenos, antibióticos y genes de resistencia 
a antibióticos (ARG) en el estiércol (Soliman et al., 2018), se ha encontrado que la eficacia de este proceso es cuestionable, puesto que es posible que la técnica no siempre sea suficiente para garantizar la inactivación completa de los patógenos y la reducción de los ARG dentro de toda la masa de compost (Castañeda-Salazar et al., 2018; Agostinho et al., 2020). Además, existe otra preocupación como es la presencia de los metales pesados, los cuales no se degradan y / o eliminan, por el contrario pueden concentrarse durante el proceso de compostaje, lo que representa una presión de selección bacteriana a largo plazo (Yang et al., 2017).

Es importante resaltar que un tratamiento deficiente de este material podría comprometer la salud y bienestar de los animales, por consiguiente es un riesgo para la salud pública; ejemplo de ello es la contaminación cruzada de las canales en el momento del beneficio, donde animales que han sido criados en camas contaminadas por bacterias causantes de ETA pueden ser fuente de transmisión para los seres humanos, ya que la cama interfiere en la composición de la microbiota gastrointestinal de las aves (Wang et al., 2016).

Por esta razón, un buen tratamiento de las camas es vital para reciclar grandes cantidades de desechos orgánicos que son una fuente importante de fertilizantes para uso agrícola (De Melo et al., 2019). En este sentido, con el fin de disminuir olores desagradables, niveles de amoniaco y carga de patógenos en las camas, son utilizados procedimientos químicos, físicos y biológicos (Kim et al., 2012; Pereira, 2016; Thomas et al., 2019).

Tratamientos químicos. Diversos tratamientos químicos modifican el pH de la cama, entre los que se encuentran; el sulfato de aluminio el cual presenta actividad inhibitoria del crecimiento de Escherichia coli, Salmonella spp. y Campylobacter spp. (Arsi et al., 2017) y la cal viva o cal hidratada, que se utiliza para desinfectar y estabilizar la cama antes del ingreso de las aves al galpón, debido a que reduce la cantidad de agua libre y además eleva el pH por encima de los rangos de tolerancia de la mayoría de los patógenos entéricos (Saraiva et al., 2019). Aunque los tratamientos químicos son recomendados para la reducción de patógenos en cama de aves, se debe tener en cuenta que la microbiota interfiere con la eficacia de estos compuestos haciendo que este tipo de tratamientos a grande escala sea poco rentable (Chen and Jiang, 2014).

Tratamientos físicos. Son aconsejados para reducir o eliminar patógenos en los desechos animales y los principales factores que deben ser considerados para que sean eficientes son: temperatura, duración del proceso y grado de humedad (Kim et al., 2012; Arsi et al., 2017; Thomas et al., 2019). Para producir un producto tratado con calor que arroje un resultado negativo o menor del límite de detección para Salmonella spp. en cama de pollo, el rango de temperatura debe ser de 65 a $80^{\circ} \mathrm{C}$ durante 30 a 60 minutos (Kim et al., 2012).

El calor seco con compostaje o sin compostaje es uno de los métodos más empleados, sin embargo, algunos patógenos pueden soportar altas temperaturas, como Salmonella Typhimurium adaptada a la desecación en cama de pollo de engorde, gracias a la activación de los genes rpoS (regulador de la respuesta de estrés general), proV (osmoprotector), dnaK (proteína chaperona) y grpE ( proteínas de choque térmico) (Chen and Jiang, 2017), otro mecanismo de resistencia a condiciones adversas en desechos animales de este patógeno es su entrada en estado viable no cultivable, lo que representa un riesgo para la salud humana ya que las bacterias conservan su potencial infectivo (Chen et al., 2018).

Tratamientos biológicos. Con el fin de acelerar procesos de compostaje, disminuir niveles de amoniaco y malos olores en camas en granjas avícolas, se utilizan microorganismos eficientes como bacterias ácido láctico, levaduras, hongos y actinomicetos, microorganismos inocuos para los seres humanos y los animales (Pereira, 2016).

Compostaje. Proceso biológico mediante el cual los microorganismos trasforman la materia orgánica biodegradable en productos estables similares al humus, donde se genera calor el cual inactiva los patógenos de origen animal presentes en estos residuos (Chen et al., 2018). Su efectividad depende de la temperatura, suministro de oxígeno, contenido de humedad, $\mathrm{pH}$, relación carbono/nitrógeno $(\mathrm{C} / \mathrm{N})$, tamaño de partícula, grado de compactación y microbiota autóctona (Kim et al., 2012; Paterlini et al., 2017). Involucra tres fases: mesófila, termófila (etapa de compostaje activo, llega a una temperatura óptima de $60^{\circ} \mathrm{C}$, donde los patógenos entéricos gene- 
ralmente son inactivados) y una fase de curación donde se da una disminución del calor hasta lograr una temperatura ambiente al final del proceso (Ren et al., 2018); la humedad optima en estas fases es de 40-60\%, vale resaltar que humedades muy altas (> $70 \%$ ) o bajas (< $40 \%$ ) pueden desacelerar o inhibir el proceso biológico (Singh and Kalamdhad, 2015).

Aunque el producto final del compostaje es considerado como un buen fertilizante, de alta calidad y seguro, prácticas de manejo inapropiadas, como no volcar las pilas, no usar cubiertas aislantes para evitar disparidades en la estratificación de la temperatura, pueden conducir a la supervivencia de patógenos (Thomas et al., 2019). Finalmente, después de este proceso es importante tener en cuenta las condiciones y tiempo de almacenamiento del material antes de ser utilizado como enmienda, debido a que periodos largos, relación carbono/ nitrógeno, propiedades fisicoquímicas y microflora adaptada del compost, afectan la inactivación térmica de patógenos (Kim et al., 2012; Chen et al., 2018).

\section{RECOMENDACIONES}

Es recomendable realizar análisis microbiológicos a las camas de aves antes, durante y después del ciclo de producción para asegurar la salud de las aves, medio ambiente, animales y humanos. No se debe utilizar gallinaza o pollinaza fresca sin tratamiento, ya que esta práctica contribuye a la diseminación de residuos nocivos, antibióticos, patógenos y sus genes de resistencia. Por otro parte, en Colombia son necesarios estudios que evalúen la supervivencia de patógenos en camas de aves con materiales como viruta de madera y cascarilla de arroz, igualmente en fertilizantes orgánicos derivados de la avicultura con el fin de evaluar el riesgo del uso de estos materiales ya que la mayoría de estudios son en países desarrollados y con diferentes condiciones climáticas.

\section{CONCLUSIONES}

En conclusión, con base a la importancia que tiene la cama de aves de corral como fuente indirecta de patógenos y residuos nocivos para los humanos, independientemente del material utilizado para este fin, es necesario realizar procesos adecuados, enfocados al manejo de olores, residuos de antibióticos, patógenos zoonóticos o de transmisión alimentar, que puedan causar problemas de salud pública y de seguridad alimentaria. Además, en Colombia existe la necesidad de generar normas para regular los límites de residuos de antibióticos y metales pesados permitidos en desechos avícolas, datos que ayudaran a buscar estrategias para el control de la cama y con ello poder direccionar cual es el uso que se le puede dar después de la cría de aves o como debe ser su disposición final.

\section{AGRADECIMIENTOS}

Los autores agradecen a la oficina de investigaciones de la Universidad del Tolima, Minciencias y a la Gobernación del Tolima, por la financiación de la investigación.

\section{CONFLICTO DE INTERESES}

No se declara ningún conflicto de interés 


\section{FINANCIACIÓN}

La presente investigación fue financiada por la convocatoria para la formación de capital humano de alto nivel para el departamento del Tolima número 755 de 2016 II-Cohorte.

\section{REFERENCIAS}

AGOSTINHO, J.M.A.; CARDOZO, M.V.; BORZI, M.M.; MARIN, J.M. Antibiotic resistance and virulence factors among Escherichia coli isolates from avian organic fertilizer. Ciência Rural, v. 50, n. 2, 2020, e 20180849. https://doi.org/10.1590/0103-8478cr20180849

AHMED, M.F.M.; SCHULZ, J; HARTUNG, J. Survival of Campylobacter jejuni in naturally land artificially contaminated laying hen feces. Poultry Science, v. 92, 2013, p. 364-369. https://doi.org/10.3382/ps.2012-02496

ANDOH, L.A.; AHMED, S.; OLSEN, J.E.; OBIRI-DANSO, K.; NEWMAN, M.J.; OPINTAN, J.A.; DALS-GAARD, A. Prevalence and characterization of Salmonella among humans in Ghana. Tropical medicine and health, $v$. 45, n. 1, 2017, p. 1-11. https://doi.org/10.1186/s41182-017-0043-z

ARSI, K.; MOORE, P.A.; DONOGHUE, A.M.; DIRAIN, M.L.; DONOGHUE, D.J. Litter Treatment With Aluminum Sulfate (Alum) Produced an Inconsistent Reduction in Horizontal Transmission of Campylobacter in Chickens. International Journal of Poultry Science, v. 16, 2017, p. 31-36. https://doi.org/10.3923/ijps.2017.31.36

BELL-COHN, A.; MAZUR, D.J.; HALL, C.; SCHAEFFER, A.J.; THUMBI-KAT, P. Uropathogenic Escherichia coli-induced fibrosis, leading to lower urinary tract symptoms, is associated with type 2 cytokine signaling. American Journal of Physiology-Renal Physiology, v. 316, n. 4, 2019, F682-F692. https://doi.org/10.1152/ajprenal.00222.2018

[7] BISWAS, S.; NAZMI, A.; PITESKY, M.; GALLARDO, R.; PANDEY, P. Thermal Inactivation of Escherichia coli and Salmonella Typhimurium in Poultry Carcass and Litter at Thermophilic Temperatures. The Journal of Applied Poultry Research, v .28, n. 2, 2019, p. 307-317. https://doi.org/10.3382/japr/pfy072

BOLAN, N.S.; SZOGI, A.A.; CHUASAVA-THI, T.; SESHADRI, B.; ROTHROCK, M.J.; PANNERSEL-VAM, P. Uses and management of poultry litter. World's Poultry Science Journal, v. 66, n. 4, 2010, p. 673-698. https://doi.org/10.1017/S0043933910000656

BROOKS, J.P.; MCLAUGHLIN, M.R.; ADELI, A.; MILES, D.M. Cultivation and Qpcr detection of pathogenic and antibiotic - resistant bacterial establishment in naive broiler houses. Journal of Environmental Quality, v. 45,2016 , p. $958-966$ https://doi.org/10.2134/jeq2015.09.0492

BUITRAGO, J.D.R.; SUÁREZ, M.C.; URIBE, C. Susceptibilidad antimicrobiana in vitro de cepas de Salmonella spp. en granjas de ponedoras comerciales del departamento de Antioquia. Revista Colombiana de Ciencias Pecuarias, v. 19, n. 3, 2006, p. 297-305.

CARMONA, F. Presencia de Campylobacter jejuni en aves de corral y sus manipuladores. Biomédica, v. 5, 1985, p. 78-85. https://doi.org/10.7705/biomedica.v5i3-4.1905

CASTAÑEDA-SALAZAR, R.; PEREIRA-BAZURDO, A.N.; DEL PILAR-PULIDO-VILLAMARIN, A.; MENDOZAGÓMEZ, M.F. Estimación de la prevalencia de Salmonella spp. en pechugas de pollo para consumo humano provenientes de cuatro localidades de Bogotá, Colombia. Infection, v. 23, 2018, p. 27-32. http://dx.doi.org/10.22354/in.v23i1.752

CASTRO-VARGAS, R.; DE RUBIO, L.F.; VEGA, A.; RONDON-BARRAGAN, I. Phenotypic and Genotypic Resistance of Salmonella Heidelberg Isolated From One of the Largest Poultry Production Region from Colombia. Poultry Science, v. 18, n. 12, 2019, p. 610-617. http://dx.doi.org/10.3923/ijps.2019.610.617 
CHEN, Z.; JIANG, X. Microbiological Safety of Chicken Litter or Chicken Litter Based Organic Fertilizers. Agriculture, v. 4, 2014, p. 1-29. http://dx.doi.org/10.3390/agriculture4010001

CHEN, Z.; DIAO, J.; DHARMASENA, M.; IONITA, C.; JIANG, X.; RIECK, J. Thermal inactivation of desiccation adapted Salmonella spp. in aged chicken litter. Applied and environmental microbiology, v. 79, n. 22, 2013, p. 7013-7020. http://dx.doi.org/10.1128/AEM.01969-13

CHEN, Z.; JIANG, X. Selection of indigenous indicator organisms for validating desiccation adapted Salmonella reduction in physically heat-treated poultry litter. Journal of applied microbiology, v. 122, n. 6, 2017, p. 1558-1569. http://dx.doi.org/10.1111/jam.13464

CHEN, Z.; KIM, J.; JIANG, X. Survival of Escherichia coli O157: H7 and Salmonella enterica in animal waste based composts as influenced by compost type, storage conation and inoculum level. Journal of applied microbiology, v. 124, n. 5, 2018, p. 1311-1323. https://doi.org/10.1111/jam.13719

COLOMBIA. INSTITUTO NACIONAL DE SALUD (INS). Informe del Evento Enfermedades Transmitidas por Alimentos, Hasta el Periodo Epidemiológico 12 del año 2017. https://www.ins.gov.co/buscadoreventos/ Informesdeevento/ETA\%202017.pdf [citado 2 de noviembre 2020]

COLOMBIA. INSTITUTO NACIONAL DE SALUD (INS). Informe del Evento Enfermedades Transmitidas por Alimentos, Hasta el Periodo Epidemiológico 12 del año 2018. https://www.ins.gov.co/buscadoreventos/ Informesdeevento/buscador\%20eventos/Informesdeevento/ENFERMEDAD\%20TRANSMITIDA\%20 POR\%20ALIMENTOS_2018.pdf [citado 2 de noviembre 2020]

COLOMBIA. INSTITUTO NACIONAL DE SALUD (INS). Informe del Evento Enfermedades Transmitidas por Alimentos, Hasta el Periodo Epidemiológico 12 del año 2019. https://www.ins.gov.co/buscadoreventos/ Informesdeevento/ENFER\%20MEDAD\%20TRANSMITIDA\%20POR\%20ALIMENTOS\%20 SEMESTRE\%20I\%202019.pdf [citado 2 de no viembre 2020]

COLOMBIA. MINISTERIO DE AMBIENTE Y DESARROLLO SOSTENIBLE. Resolución 1541. (Noviembre 12 de 2013). Por la cual se establecen los niveles permisibles de calidad del aire o de inmisión, el procedimiento para la evaluación de actividades que generan olores ofensivos y se dictan otras disposiciones. Diario Oficial. Bogotá (Colombia): 2013.

COLOMBIA. FEDERACIÓN NACIONAL DE AVICULTORES (FENAVI). Boletín fenaviquín número 278. 2019. https://fenavi.org/wpcontent/uploads/2018/12/Fenaviquin_ed 2772018-2. pdf [citado 3 de enero 2020]

CONESA, J.A. Dioxins from Agro Waste Combustion: Evaluation and Management. Byproducts from Agriculture and Fisheries: Adding Value for Food, Feed. Pharma and Fuels, 2019, p. 629-640. https://doi.org/10.1002/9781119383956.ch28

DAHSHAN, H.; MERWAD, A.M.; MOHAMED, T.S. Listeria species in broiler poultry farms: Potential public health hazards. Journal of microbiology and biotechnology, v. 26, 2016, p. 1551-1556. https://doi.org/10.4014/jmb.1603.03075

DE CARVALHO, G.B.; SANTOS, N.; MARTINS, J.D.S.; PEREIRA, N.M.; FALLEIROS, M.B.; ARNHOLD, E.; CAFÉ, M.B. Litter quality of broiler fed with to different levels of sulfur amino acid. Journal of Animal Behaviour and Biometeorology, v. 6, n. 1, 2018, p. 21-28.

https://doi.org/10.14269/2318-1265/jabb.v6n1p21-28

DE MELO, T.R.; FIGUEIREDO, A.; MACHADO, W.; TAVARES-FILHO, J. Changes on soil structural stability after in natura and composted chicken manure application. International Journal of Recycling of Organic Waste in Agriculture, v. 8, n. 4, 2019, p. 333-338.

https://doi.org/10.1007/s40093-019-0250-1

DONADO-GODOY, P.; GARDNER, I.; BYRNE, B.A.; LEON, M.; PEREZ-GUTIERREZ, E.; OVALLE, M.V.; TAFUR, M.A.; MILLER, W. Prevalence, risk factors, and antimicrobial resistance profiles of Salmonella from commercial broiler farms in two important poultry-producing regions of Colombia. Journal of food protection, v. 75, n. 5, 2012, p. 874-883.

https://doi.org/10.4315/0362-028X.JFP-11-458 
DUNLOP, M.W.; BLACKALL, P.J.; STUETZ, R.M. Odour emissions from poultry litter - A review litter properties, odour formation and odorant emissions from porous materials. Journal of Environmental Management, v. 177, 2016, p. 306-319. https://doi.org/10.1016/j.jenvman.2016.04.009

FANDIÑO, L.C.; VERJAN-GARCÍA, N. A common Salmonella Enteritidis sequence type from poultry and human gastroenteritis in Ibague, Colombia. Biomédica, v. 39, 2019, p. 50-62. http://dx.doi.org/10.7705/biomedica.v39i1.4155

FANG, H.; WANG, H.; CAI, L.; YUNLONG, Y. Prevalence of antibiotic resistance genes and bacterial pathogens in long term manured greenhouse soils as revealed by metagenomic survey. Environmental science \& technology, v. 49, 2015, p. 1095-1104. http://dx.doi.org/10.1021/es504157v

FARHADI, D. Evaluation of the physical and chemical properties of some agricultural wastes as poultry litter material. Global Journal of Animal Scientific Research, v. 2, 2014, p. 270-276.

GÓMEZ, L.F.; TORRES, I.P.; MCEWEN, J.G.; DE BEDOUT, C.; PELÁEZ, C.A.; ACEVEDO, J.M.; TAYLOR, M.L.; ARANGO, M. Detection of Histoplasma capsulatum in Organic Fertilizers by Hc100 Nested Polymerase Chain Reaction and Its Correlation with the Physicochemical and Microbiological Characteristics of the Samples. The American journal of tropical medicine and hygiene, v. 98, n. 5, 2018, p. 1303-1312. http://dx.doi.org/10.4269/ajtmh.17-0214

GONZALEZ, R.M.; ANGELES, H.J.C. Antibiotic and synthetic growth promoters in animal diets: Review of impact and analytical methods. Food Control, v. 72, 2017, p. 255-267. http://dx.doi.org/10.1016/j.foodcont.2016.03.001

GUAN, J.; CHAN, M.; GRENIER, C.; WILKIE, D.C.; BROOKS, B.W.; SPENCER, J.L. Survival of avian influenza and Newcastle disease viruses in compost and at ambient temperatures based on virus isolation and real-time reverse transcriptase PCR. Avian diseases, v. 53, n. 1, 2009, p. 26-33. http://dx.doi.org/10.1637/8381-062008-Reg.1

IBRAHIM, M.A.; EMEASH, H.H.; GHONEIM, N.H.; ABDEL-HALIM, M.A. Seroepidemiological Studies on Poultry Salmonellosis and its Public Health Importance. Journal of World's Poultry Research, v. 3, 2013, p.18-23.

ISLAM, M.J.; SULTANA, S.; DAS, K.K.; SHARMIN, N.; HASAN, M.N. Isolation of plasmid mediated multidrug resistant Escherichia coli from poultry. International Journal of Sustainable Crop Production, v. 3, n. 5 , 2008, p. 46-50.

KAGAMBĖGA, A.; THIBODEAU,A.;TRINETTA, V.; SORO, D.K.;SAMA, F.N.;BAKO, C.S.; NDIAYE, A.W.; FRAVALO, P.; BARRO, N. Salmonella spp. and Campylobacter spp. In poultry feces and carcasses in Ouagadougou, Burkina Faso. Food Science \& nutrition, v. 6, 2018, p. 1601-1606. https://doi.org/10.1002/fsn3.725

KANDUN, I.N.; SAMAAN, G.; HARUN, S.; PURBA, W.H.; SARIWATI, E.; SEPTIAWATI, C.; WANDRA, T. Chicken faeces garden fertilizer: possible source of human avian influenza H5N1 infection. Zoonoses Public Health, v. 57, 2010, p. 285-290. http://dx.doi.org/10.1111/j.1863-2378.2009.01246.x

KASSEM, I.I.;SANAD, Y.; GANGAIAH, D.; LILBURN, M.; LEJEUNE, J.; RAJASHE-KARA, G. Use of bioluminescence imaging to monitor Campylobacter survival in chicken litter. Journal of applied microbiology, 2010, v. 109, p. 1988-1997. http://dx.doi.org/10.1111/j.1365-2672.2010.04828.x

KASSEM, I.I.; KEHINDE, O.; KUMAR, A.; RAJASHEKARA, G. Antimicrobial al resistant Campylobacter in organically and conventionally raised layer chickens. Foodborne pathogens and disease, v. 14, n. 1, 2017 , p. 29-34. http://dx.doi.org/10.1089/fpd.2016.2161

KIM, J.; DIAO, J.; SHEPHERD, M.W.; SINGH, R.; HERINGA, S.D.; GONG, C.; JIANG, X. Validating thermal inactivation of Salmonella spp. in fresh and aged chicken litter. Applied and environmental microbiology, v. 78, 2012, p. 1302-1307.

http://dx.doi.org/10.1128/AEM.06671-11 
KOBAYASHI, S.D.; MALACHOWA, N.; DELEO, F.R. Pathogenesis of Staphylococcus aureus abscesses. The American journal of pathology, v. 185, n. 6, 2015, p.1518-1527. http://dx.doi.org/10.1016/j.ajpath.2014.11.030

KUMAR, K.; THOMPSON, A.; SINGH, A.K.; CHANDER, Y.; GUPTA, S. Enzyme-linked immunosorbent assay for ultrace determination of antibiotics in aqueous samples. Journal of Environ mental Quality, v. 33, n. 1, 2004, p. 250-256. http://dx.doi.org/10.2134/jeq2004.0250

KYAKUWAIRE, M.; OLUPOT, G.; AMODING, A.; NKEDI-KIZZA, P.; ATEENYI BASAMBA, T. How Safe is Chicken Litter for Land Application as an Organic Fertilizer?: A Review. International journal of environmental research and public health, v. 16, n. 19, 2019, p. 3521-3544. http://dx.doi.org/10.3390/ijerph16193521

LABBE, R.G.; JUNEJA, V.K. En: Clostridium perfringens. In Foodborne diseases. 3 ed. New York (United States Of America): Elsevier Inc, 2017, p. 235-242.

LI, X.; PAYNE, J.B.; SANTOS, F.B.; LEVINE, J.F.; ANDERSON, K.E.; SHELDON, B.W. Salmonella populations and prevalence in layer feces from commercial highrise houses and characterization of the Salmonella isolates by serotyping, antibiotic resistance ana- lysis, and pulsed field gel electrophoresis. Poultry science, v. 86, n. 3, 2007, p. 591-597. http://dx.doi.org/10.1093/ps/86.3.591

LIU, H.Q.; ZENG, T.T.; WEI, G.X.; ZHANG, R.; LIU, F.; WANG, H. Comparison of dioxin destruction in the fly ash and froths under microwave irradiation. Aerosol and Air Quality Research, v. 19, 2019, p. 925-936. http://dx.doi.org/10.4209/aaqr.2018.09.0337

MARTÍNEZ-HERNÁNDEZ, L.; CARO-SÁNCHEZ, C.H.; BONIFAZ, A. Infecciones por Fusarium. Dermatologia Revista Mexicana, v. 58, n. 5, 2014, p. 432-442.

MILANOV,D.; KNEŽEVIĆ, S.; VIDAKO-VIĆ, S.; PAJIĆ, M.; ŽIVKOV-BALOŠ; ALEKSIĆ, N. Microbial contamination of poultry litter during fattening period. Biotechnology in Animal Husbandry, v. 35, n. 3, 2019, p. 253-265. http://dx.doi.org/10.2298/BAH1903253M

NANDI, S.; MAURER, J.J.; HOFACRE, C.; SUMMERS, A.O. Gram-positive bacteria are a major reservoir of Class 1 antibiotic resistance integrons in poultry litter. Proceedings of the National Academy of Sciences, v. 101, n.18, 2004, p. 7118-7122. http://dx.doi.org/10.1073/pnas.0306466101

NGAJILO, D.; SINGH, T.; RATS-HIKHOPHA, E.; DAYAL, P.; MATUKA, O.; BAATJIES, R.; JEEBHAY M.F. Risk factors associated with allergic sensitization and asthma phenotypes among poultry farm workers. American journal of industrial medicine, v. 61, 2018, p. 515-523. http://dx.doi.org/10.1002/ajim.22841

ORGANIZACIÓN MUNDIAL DE LA SALUD (OMS). Resistencia antimicrobiana, reporte global en vigilancia. Ginebra. 2014. https://www.who.int/mediacentre/news/releases/2014/amr-report/es/ [citado 19 de octubre 2019]

PATERLINI, H.; GONZÁLEZ, V.; PICONE, L.I. Calidad de la cama de pollo fresca y compostada. Ciencia del suelo, v. 35, n. 1, 2017, p. 69-78.

PEREIRA-PEÑATE, N. Uso de microorganismos eficientes (M.E) en pollinaza para disminuir los niveles de amoniaco (NH3) en granjas avícolas comerciales de Sincelejo, Colombia. Revista Colombiana de Ciencia Animal RECIA, v. 8, 2016, p. 386-390. http://dx.doi.org/10.24188/recia.v8.n0.2016.395

PIGNATA-VIANA, M.C.; SANTOS, J.S.; VIANA, P.T. Epidemiologia e fatores de riscos relacionados à intoxicação alimentar causada por Clostridium Botulinum: uma revisão narrativa. Clinical \& Biomedical Research, v. 39, n. 2, 2019, p. 161-170. http://dx.doi.org/10.4322/2357-9730.85961

PREMARATHNE, J.M.K.J.K; SATHA-RASINGHE, D.A; HUAT, J.T.Y; BAS-RI, D.F; RUKAYADI, Y.; NAKAGUCHI, Y.; NISHIBUCHI, M.; RADU, S. Impact of human Campylobacter infections in Southeast Asia: The contribution of the poultry sector. Critical reviews in food science and nutrition, v. 57, 2017, p. 3971-3986.

http://dx.doi.org/10.1080/10408398.2016.1266297 
PULIDO-LANDÍNEZ, M.; SÁNCHEZ-INGUNZA, R.; GUARD, J.; DONASCIMENTO, V.P. Presence of Salmonella Enteritidis and Salmonella gallinarum in commercial laying hens diagnosed with fowl typhoid disease in Colombia. Avian diseases, v. 58, n. 1, 2014, p. 165-170. http://dx.doi.org/10.1637/10598-062613-Case.1

RADOSHEVICH, L.; COSSART, P. Listeria monocytogenes: towards a complete picture of its physiology and pathogenesis. Nature Reviews Microbiology, v. 16, n. 1, 2017, p. 32-46. http://dx.doi.org/10.1038/nrmicro.2017.126

RAMÍREZ-HOBAK, L.; GÓMEZ-SÁENZ, A.; VEGA-SÁNCHEZ, D.C.; ARENAS, R. Onicomicosis por mohos no dermatofitos: Una revisión. Dermatología Cosmética, Médica y Quirúrgica, v. 15, n. 3, 2017, p. 184-195.

REN, X.; ZENG, G.; TANG, L.; WANG, J.; WAN, J.; WANG, J.; DENG, Y.L.; PENG, B. The potential impact on the biodegradation of organic pollutants from composting technology for soil remediation. Waste management, v. 72, 2018, p. 138-149. http://dx.doi.org/10.1016/j.wasman.2017.11.032

RIDDELL, J.; WHEAT, L.J. Central Nervous System Infection with Histoplasma capsulatum. Journal of Fungi, v. 5, n. 3, 2019, p. 60-70. http://dx.doi.org/10.3390/jof5030070

RITZ, C.W.; FAIRCHILD, B.D.; LACY, M.P. Litter Quality and Broiler Performance. Cooperative Extension, University of Georgia (Bulletin 1267). 2009. https://extension.uga.edu/publications/detail. html?number=B1267 [citado 19 de octubre 2019]

RODRÍGUEZ, R.; FANDIÑO, C.; DONADO, P.; GUZMÁN, L.; VERJAN, N. Characterization of Salmonella from commercial egg-laying hen farms in a central region of Colombia. Avian diseases, v. 59, n. 1, 2015, p. 57-63. http://dx.doi.org/10.1637/10873-052714-reg

RODRIGUEZ, J.M.; RONDÓN, I.S.; VERJAN, N. Serotypes of Salmonella in Broiler Carcasses Marketed at Ibague, Colombia. Brazilian Journal of Poultry Science, v. 17, n. 4, 2015, p. 545-552. http://dx.doi.org/10.1590/1516-635x1704545-552

RYAN, U.; ZAHEDI, A.; PAPARINI, A. Cryptosporidium in humans and animals a one health approach to prophylaxis. Parasite Immunology, v. 38, n. 9, 2016, p. 535-547. http://dx.doi.org/10.1111/pim.12350

SARAIVA, M.M.S.; MOREIRA-FILHO, A.L.B.; VASCONCELOS, P.C.; NASCIMENTO, P.V.; AZEVEDO, P.S.; NETO, O.F.; GIVISIEZ, P.E.N.; GEBREYES, W.A.; OLIVEIRA, C.J.B. Chemical treatment of poultry litter affects the conjugation of plasmid mediated extended spectrum betalactamase resistance genes in E. coli. Journal of Applied Poultry Research, v. 29, 2019, p. 197-203. https://doi.org/10.1016/j.japr.2019.10.006

SHAH, M.K.; BRADSHAW, R.; NYARKO, E.; HANDY, E.T.; EAST, C.; MILLNER, P.D.; BERGHOL, T.M.; SHARMA, M. Salmonella enterica in soils amended with heat-treated poultry pellets survived longer than bacteria in unamended soils and more readily transferred to and persisted on spinach. Applied and environmental microbiology, v. 85, n. 10, 2019, e 00334-19. http://dx.doi.org/10.1128/AEM.00334-19

SHECHO, M.; THOMAS, N.; KEMAL, J.; MUKTAR, Y. Cloacael carriage and multidrug resistance Escherichia coli 0157: H7 from poultry farms, eastern Ethiopia. Journal of veterinary medicine, 2017, p. 1-19. http://dx.doi.org/10.1155/2017/8264583

SHEFFIELD, C.L.; CRIPPEN, T.L.; BEIER, R.C. Multimicrobial compounds eliminate or reduce Salmonella typhimurium from one-third of poultry liter samples within 8 days. Research Journal of Poultry Sciences, $v$. 11, n. 1, 2018, p. 5-8. http://dx.doi.org/10.3923/rjpscience.2018.5.8

SILLER, P.; DAEHRE, K.; THIEL, N.; NÜBEL, U.; ROESLER, U. Impact of short-term storage on the quantity of extended spectrum betalactamase proproducing Escherichia coli in broiler litter under practical conditions. Poultry science, v. 99, n. 4, 2020, p. 2125-2135.

https://doi.org/10.1016/j.psj.2019.11.043 
SINGH, J.; KALAMDHAD, A.S. Assessment of compost quality in agitated pile composting of water hyacinth collected from different sources. International Journal of Recycling of Organic Waste in Agriculture, v. 4, n. 3, 2015, p. 175-183. http://dx.doi.org/10.1007/s40093-015-0097-z

SMITH, S.; MEADE, J.; GIBBONS, J.; MCGILL, K.; BOLTON, D.; WHYTE, P. The impact of environmental conditions on Campylobacter jejuni survival in broiler faeces and litter. Infection ecology \& epidemiology, v. 6, n. 1, 2016, p. $31685-31692$. http://dx.doi.org/10.3402/iee.v6.31685

SMITH, T.C. Livestock-associated Staphylococcus aureus: the United States experience. Plos Pathog, v. 11, 2015, e 1004564. http://dx.doi.org/10.1371/journal.ppat.1004564

SOLIMAN, E.S; SALLAM, N.H.; ABOUELHASSAN, E.M. Effectiveness of poultry litter amendmen ts on bacterial survival and Eimeria oocyst sporulation. Veterinary world, v. 11, n. 8, 2018, p. 1064-1073. http://dx.doi.org/10.14202/vetworld.2018.1064-1073

STOJCIC, M.Đ.; BJEDOV, S.; ŽIKIC, D.; PERIC, L.; MILOŠEVIC, N. Effect of straw size and microbial amendment of litter on certain litter quality parameters, ammonia emission, and footpad dermatitis in broilers. Archives Animal Breeding, v. 59, 2016, p. 131-137. http://dx.doi.org/10.5194/aab-59-131-2016

THOMAS, C.; IDLER, C.; AMMON, C.; HERRMANN, C.; AMON, T. Inactivation of ESBL /Ampc producing Escherichia coli during mesophilic and thermophilic anaerobic digestion of chicken manure. Waste managenet, v. 84, 2019, p. 74-82. http://dx.doi.org/10.1016/j.wasman.2018.11.028

TUALEKA, A.R.; FARADISHA, J.; MAHARJA, R. Determination of No Observed - Adverse effect Level Ammonia in White Mice Through CD4 Expression. Dose-Response, v. 16, 2018, p. 1559325818807790. http://dx.doi.org/10.1177/15593 25818807790.

VAINIO-KAILA, T.; ZHANG, X.; HÄNNINEN, T.; KYYHKYNEN, A.; JOHASSON, L.S.; WILLFÖR, S.; ÖSTERBERG, M.; SIITONEN, A.; RAUTKARI, L. Antibacterial effects of wood structural components and extractives from Pinus sylvestris and Picea abies on Methicillin-Resistant Staphylococcus aureus and Escherichia coli O157:H7. BioReources, v. 12, 2017, p. 7601-7614. http://dx.doi.org/10.15376/biores.12.4.7601-7614

VAZ, C.S.L.; VOSS-RECH, D.; DE AVILA, V.S.; COLDEBELLA, A.; SILVA, V.S. Interventions to reduce the bacterial load in recycled broiler litter. Poultry science, v. 96, n. 8, 2017, p. 2587-2594. https://doi.org/10.3382/ps/pex063

VERMEULEN, L.C.; BENDERS, J.; MEDEMA, G.; HOFSTRA, N. Global Cryptosporidium loads from livestock manure. Environmental science \& technology, v. 51, n. 15, 2017, p. 8663-8671. http://dx.doi.org/10.1021/acs.est.7b00452

VIEGAS, S.; VEIGA, L.; ALMEIDA, A.; DOS SANTOS, M.; CAROLINO, E.; VIEGAS, C. Occupational exposure to aflatoxin B1 in a Portuguese poultry slaughterhouse. Annals of Occupational Hygiene, v. 60, n. 2, 2015, p.176-183. http://dx.doi.org/10.1093/annhyg/mev077

WANG, L.; LILBURN, M.; ZHONG-TANG, Y. Intestinal microbiota of broiler Chicken as affected by litter manageme regimen. Frontiers in Microbiology, v. 7, 2016, p.1-12.10.3389/fmicb.2016.00593

XIE, W.; SHEN, Q.; ZHAO, F.J. Antibiotics and antibiotic resistance from animal manures to soil: a review. European journal of soil science, v. 69, n. 1, 2018, p. 181-195. http://dx.doi.org/10.1111/ejss.12494

XIONG, W.; WANG, M.; DAI, J.; SUN, Y.; ZENG, Z. Application of manure containing tetracyclines slowed down the dissipation of tet resistance genes and caused changes in the composition of soil bacteria. Ecotoxicology and environmental safety, v. 147, 2018, p. 455-460. http://dx.doi.org/10.1016/j.ecoenv.2017.08.061 
YAMAZAKI,W.; UEMURA, R.;SEKIGUCHI,S.; DONG, J.B.; WATANABE,S.; KIRINO, Y.; MISAWA, N. Campylobacter and Salmonella are prevalent in broiler farms in Kyushu, Japan: Results of a 2-year distribution and circulation dynamics audit. Journal of Applied Microbiology, v. 120, n. 6, 2016, p. 1711-1722. https://doi.org/10.1111/jam.13141

YANG, X.; LI, Q.; TANG, Z.; ZHANG, W.; YU, G.; SHEN, Q.; ZHAO, F.J. Heavy metal concentrations and arsenic speciation in animal manure composts in China. Waste Management, v. 64, 2017, p. 333-339. https://doi.org/10.1016/j.wasman.2017.03.015 